\section{Insegurança alimentar como indicador de iniqüidade: análise de inquérito populacional}

\author{
Food insecurity as an indicator of inequity: \\ analysis of a population survey
}

\footnotetext{
${ }^{1}$ Faculdade de Ciências Médicas, Universidade Estadual de Campinas, Campinas, Brasil.

2 University of Connecticut, Storrs, U.S.A.

Correspondência G. Panigassi Departamento de Medicina Preventiva e Social, Faculdade de Ciências Médicas, Universidade Estadual de Campinas. Rua Tessália Vieira de Camargo 126, Cidade Universitária-Barão Geraldo, Campinas, $S P$ 13081-970, Brasil. panigi@unicamp.br
}

\begin{abstract}
This study aimed to analyze the association between food insecurity and certain socioeconomic and demographic variables that measure social inequality: income, schooling, race, family structure, household characteristics, and sewage conditions. A sample of 456 families in Campinas, São Paulo State, was interviewed using the Brazilian Food Insecurity Scale (EBIA). Family food insecurity was associated with: more children < 18 years; precarious housing; overcrowding; lack of sewage system; low income $(<2$ times the minimum wage); head-of-household with no schooling; no university graduate in the family; and race (black). Directly measuring food insecurity is important for monitoring inequality, and can be used either with other socioeconomic and demographic indicators or alone to identify social vulnerability in population groups.
\end{abstract}

Food Security; Social Inequity; Social Conditions

\author{
Giseli Panigassi 1 \\ Ana Maria Segall-Corrêa ${ }^{1}$ \\ Letícia Marin-León 1 \\ Rafael Pérez-Escamilla 2 \\ Maria de Fátima Archanjo Sampaio 1 \\ Lucia Kurdian Maranha 1
}

\section{Introdução}

As desigualdades sociais entre os países, medidas pelas diferenças de escolaridade, renda e longevidade, têm sido evidenciadas, e o Brasil ocupa, segundo medida decrescente do Índice de Desenvolvimento Humano (IDH), a 69a posição no ranking mundial de um total de 177 listados 1 .

Conhecer e estabelecer indicadores de avaliação da desigualdade têm sido objeto de trabalho de muitos estudiosos em diversas áreas, como educação 2, saúde ${ }^{3,4}$ e economia 5,6. Apesar da forte influência das condições econômicas sobre a polarização do perfil social da população, existem outras condições que se combinam às desigualdades e cujas representações sociais estão associadas a estigmas, de que são vítimas, como exemplos, negros, índios, mulheres, idosos e nordestinos 3 .

No Brasil, as diferenças sociais, tradicionalmente, vêm sendo monitoradas mediante variáveis sócio-econômicas, como renda, escolaridade e esperança de vida ao nascer ${ }^{1}$. O recente trabalho de validação da Escala Brasileira de Segurança Alimentar, que mede a experiência e a percepção de insegurança alimentar e fome no nível familiar, tornou disponível um indicador direto dessas condições 7 .

Considerando que a desigualdade social reflete as diferenças produzidas socialmente e que são eticamente injustas 8 , chega-se ao conceito de iniqüidade social aplicável à situação de in- 
segurança alimentar. Pode-se, dessa forma, assumir que a iniqüidade na segurança alimentar constitui-se em diferenças de acesso aos alimentos e à alimentação saudável, condições essas socialmente produzidas e que impactam negativamente no bem-estar e na qualidade de vida das famílias e de seus membros. Nesse sentido, a insegurança alimentar reflete a negação aos direitos elementares da pessoa, como os de estar vivo e sem doença, estar bem nutrido, assim como de ter garantido o seu direito ao respeito próprio e o de ser respeitado como cidadão ${ }^{9}$. Relaciona-se a diferentes problemas de saúde, como o comprometimento do desenvolvimento de crianças escolares nas habilidades de matemática, de leitura e sociais 10; a depressão materna e o comprometimento da saúde infantil 11; além disso, sintomas de estresse, ansiedade, e depressão também tem sido observados em grávidas com insegurança alimentar 12 .

Por esses motivos, justifica-se a análise do indicador de insegurança alimentar familiar como medida da condição de iniqüidade social.

Este trabalho tem o objetivo de investigar a associação da insegurança alimentar com algumas variáveis indicativas de desigualdades sociais, como renda, escolaridade, raça/cor, composição familiar, características da moradia e condições de saneamento.

\section{Métodos}

Realizou-se inquérito amostral com população constituída por famílias residentes na zona urbana do Município de Campinas, São Paulo, Brasil.

O processo de amostragem adotado foi o de conglomerados em dois estágios. Trinta setores censitários foram sorteados aleatoriamente no primeiro estágio e foram agrupados em três estratos sociais, medidos pelos percentuais de chefes de família com nível universitário em cada um deles: menos de $25 \%$ de chefes de família no setor; de $25 \%$ a $50 \%$; mais de $50 \%$. Procedeu-se à atualização do número de domicílios nesses setores com base no cadastro fornecido pela coordenação do Inquérito de Saúde no Estado de São Paulo. Foram sorteados mil domicílios, segundo a probabilidade de seleção proporcional ao tamanho do setor censitário.

Dos mil domicílios sorteados, incluíram-se nessa análise apenas aqueles em que residiam famílias com, pelo menos, um integrante menor de 18 anos de idade $(n=456)$. A amostra limitou-se a domicílios com essa característica em virtude de a Escala Brasileira de Insegurança Alimentar (EBIA) utilizar oito perguntas referentes a essa faixa etária.
O levantamento dos dados foi realizado entre os meses de julho e agosto de 2003, por meio de entrevista domiciliar direta com um membro da família, reconhecidamente conhecedor da dinâmica alimentar familiar, na forma de questionário padronizado, com perguntas que informam condições sócio-econômicas, demográficas e de segurança alimentar (EBIA) nos três últimos meses. Esse instrumento foi adaptado daquele proposto pelo Departamento de Agricultura dos Estados Unidos 13 e validado para aplicação em área urbana no Brasil 7.

A unidade de estudo foi a família, sendo classificada em três diferentes níveis de segurança alimentar - segurança alimentar, insegurança alimentar leve e insegurança alimentar moderada e grave -, conforme a somatória das respostas positivas das 15 questões do instrumento EBIA. A opção de trabalhar apenas três categorias, juntando o nível moderado com o grave, deve-se ao número reduzido de famílias em algumas estratificações de variáveis na análise dos dados.

Utilizou-se o programa Stata (versão 7.0. Stata Corporation, College Station, Estados Unidos) para a análise dos dados, pois permite o cálculo de estimativas populacionais, considerando as diferentes probabilidades de seleção dos domicílios de amostras complexas.

Considerando as devidas frações de expansão dos dados, calcularam-se as prevalências dos diferentes níveis de segurança alimentar segundo características sócio-econômicas e demográficas. Para constatar diferenças na distribuição das proporções de insegurança alimentar segundo as categorias da variável independente, foi utilizado o teste qui-quadrado, sendo a diferença considerada significativa quando o valor de $\mathrm{p}$ foi $\leq 0,05$.

A investigação das possíveis variáveis associadas à segurança/insegurança alimentar foi realizada utilizando-se os seguintes parâmetros para construção de um modelo de regressão logística politômica univariada e multivariada com os odds proporcionais: (1) variável dependente com valores de 1 para segurança alimentar; 2 para insegurança alimentar leve e 3 para insegurança alimentar moderada e grave, pois a variável resposta segue uma ordem natural 14; (2) variáveis independentes categóricas: sexo (feminino ou masculino) e escolaridade do chefe da família (agrupada em três categorias: analfabeto/fundamental incompleto, nível fundamental completo ou nível médio completo/universitário); raça/cor auto-referida do informante (preta, parda ou branca), renda mensal familiar em salário mínimo dicotomizada ( $\leq 2$ salários mínimos ou > 2 salários mínimos), membro familiar com nível universitário (sim ou não), membro familiar analfabeto (sim ou não), esgoto da re- 
de pública no domicílio (sim ou não) e tipo de construção da moradia (alvenaria acabada ou outro material); (3) variáveis independentes contínuas: número de menores de cinco anos no domicílio, número de menores de 18 anos no domicílio, número de adultos no domicílio (entre 18 e 64 anos) e número de pessoas por cômodo do domicílio.

No modelo multivariado, foram incluídas todas as variáveis que apresentaram $\mathrm{p} \leq 0,20$ na análise univariada. As variáveis independentes que apresentaram alta colinearidade, como número de menores de cinco anos e número de menores de 18 anos, esgoto da rede pública e tipo de construção, foram testadas em modelos separados, permanecendo o modelo que melhor explicou a variável dependente. Foram incluídas, ainda, no modelo multivariado, oito variáveis independentes: sexo do chefe; escolaridade do chefe; raça/cor auto-referida do informante; renda mensal familiar em salário mínimo; membro da família com nível universitário; número de menores de 18 anos no domicílio; número de pessoas por cômodo do domicílio; tipo de construção da moradia. Permaneceram no modelo final aquelas que se mostraram significativas após os procedimentos de ajuste $(\mathrm{p}<0,05)$.

Os trabalhos iniciaram-se após o recebimento do parecer favorável do Comitê de Ética em Pesquisa da Faculdade de Ciências Médicas da Universidade Estadual de Campinas e do consentimento livre e esclarecido dos participantes.

\section{Resultados}

Verificou-se, neste estudo, que a insegurança alimentar em Campinas atinge 60,5\% das famílias com menores de 18 anos de idade; esse percentual está dividido em duas formas de gravidade: 40,1\% estão em condição de insegurança alimentar leve e $20,4 \%$, de insegurança alimentar moderada e grave.

Foram encontrados, em cerca de $70 \%$ das famílias, homens como chefes; em média, os chefes tinham 43 anos de idade e $28 \%$ não completaram o ensino fundamental, sendo $5,1 \%$ analfabetos.

Em média (Tabela 1), encontraram-se 4,3 pessoas por domicílio (IC95\%: 4,127-4,376), 0,9 pessoas por cômodo (IC95\%: 0,768-0,967), 9\% de membros da família sem alfabetização e $30 \%$ de pessoas com nível universitário.

$\mathrm{Na}$ maioria das famílias entrevistadas, o informante declarou-se de cor branca (Tabela 2). Verificou-se, também, que as famílias com informantes de cor preta ou parda apresentaram prevalências significativamente maiores $(\mathrm{p}<0,0001)$ de insegurança alimentar moderada e grave, comparativamente às famílias com informantes de cor branca. Existe um gradiente decrescente e significativo da proporção de famílias em segurança alimentar, insegurança alimentar leve e insegurança alimentar moderada e grave no grupo de cor branca, enquanto no grupo de cor preta a proporção de insegurança alimentar leve foi maior que as outras duas categorias (insegurança alimentar moderada e grave e segurança alimentar), sendo muito homogêneos os percentuais das três categorias no grupo de famílias com informantes de cor parda.

Para a análise do comportamento das categorias de insegurança em relação à renda, utilizaram-se como ponto de corte dois salários mínimos da renda familiar mensal, tendo-se como valor de referência $\mathrm{R} \$ 240,00$ (agosto de 2003). A renda da família (Tabela 2) apresentou grande variabilidade, e a mediana situou-se em 4,3 salários mínimos. Foram observadas altas proporções de insegurança (insegurança alimentar leve $=44,6 \%$ e insegurança alimentar moderada e grave $=43,5 \%$ ) (Tabela 2) entre as famílias com renda menor de dois salários mínimos; entretanto, no nível superior de rendimentos, encontrou-se percentual pequeno de famílias com insegurança alimentar moderada e grave e alto percentual com insegurança alimentar leve. É interessante ressaltar a presença de aproximadamente $12 \%$ de famílias em segurança alimentar com renda abaixo de dois salários mínimos.

Foram praticamente universais a coleta de lixo e o abastecimento de água com encanamento interno e disponibilidade diária; portanto, não houve diferença significativa entre os níveis de insegurança alimentar (Tabela 2).

Os três níveis de segurança se relacionaram com outras desigualdades nesse inquérito: existem diferenças significativas em relação ao saneamento básico. Rede de esgoto encanada não estava disponível em 9,4\% dos domicílios do grupo em segurança alimentar, 16,7\% dos em insegurança alimentar leve e 35\% dos em insegurança alimentar moderada e grave. Ainda em relação às desigualdades sanitárias do domicílio, enquanto apenas $4,4 \%$ das famílias em segurança alimentar tinham esgoto a "céu aberto", entre famílias em insegurança alimentar leve essa proporção foi quatro vezes maior e entre aquelas em insegurança alimentar moderada e grave, o pior cenário: quase seis vezes mais. A referência de criança com verminose no domicílio também foi mais freqüente nas famílias em insegurança alimentar moderada e grave $(19,5 \%)$.

A associação entre insegurança alimentar e as características familiares que apresentaram significância estatística foi medida por procedimentos de regressão logística politômica univa- 
Composição familiar apresentada por categoria de segurança alimentar, insegurança alimentar leve e insegurança alimentar moderada e grave. Campinas, São Paulo, Brasil, 2003

\begin{tabular}{|c|c|c|c|c|}
\hline $\begin{array}{l}\text { Características do do domicílio } \\
\text { ou da família }\end{array}$ & Geral & $\begin{array}{l}\text { Segurança } \\
\text { alimentar }\end{array}$ & $\begin{array}{c}\text { Insegurança } \\
\text { alimentar leve }\end{array}$ & $\begin{array}{c}\text { Insegurança } \\
\text { alimentar } \\
\text { moderada e grave }\end{array}$ \\
\hline Média (desvio-padrão) do número de pessoas & $4,25(21,31)$ & $4,00(18,47)$ & $4,36(20,88)$ & $4,52(25,67)$ \\
\hline Média (desvio-padrão) do número de cômodos & $5,79(39,60)$ & $6,75(40,34)$ & $5,44(34,71)$ & $4,66(36,11)$ \\
\hline Média (desvio-padrão) do número de pessoas por cômodo & $0,87(0,50)$ & $0,67(0,43)$ & $0,91(0,35)$ & $1,15(0,78)$ \\
\hline Percentual de pessoas sem alfabetização & 9,0 & 4,0 & 9,0 & 19,0 \\
\hline Percentual de pessoas com nível universitário & 30,0 & 56,0 & 17,0 & 5,0 \\
\hline
\end{tabular}

Tabela 2

Características das famílias, de seus informantes e dos seus domicílios, apresentadas por categoria de segurança alimentar, insegurança alimentar leve e insegurança alimentar moderada e grave. Campinas, São Paulo, Brasil, 2003.

\begin{tabular}{|c|c|c|c|c|c|}
\hline Característica & Geral (\%) & $\begin{array}{l}\text { Segurança } \\
\text { alimentar }\end{array}$ & $\begin{array}{l}\text { Insegurança } \\
\text { alimentar leve }\end{array}$ & $\begin{array}{c}\text { Insegurança } \\
\text { alimentar } \\
\text { moderada e grave }\end{array}$ & Valor de $p$ * \\
\hline \multicolumn{6}{|l|}{ Raça/cor auto-referida do informante } \\
\hline Branca & 60,6 & 45,6 & 39,4 & 15,0 & 0,011 \\
\hline Parda & 29,9 & 31,0 & 35,9 & 33,1 & \\
\hline Preta & 9,5 & 18,4 & 53,8 & 27,8 & \\
\hline \multicolumn{6}{|l|}{ Renda familiar mensal (salário mínimo) ** } \\
\hline Até 2 & 35,1 & 11,9 & 44,6 & 43,5 & $<0,001$ \\
\hline Mais de 2 & 64,9 & 54,6 & 37,9 & 7,5 & \\
\hline Coleta de lixo & 99,8 & 100,0 & 99,4 & 100,0 & $>0,05$ \\
\hline Encanamento interno de água & 99,3 & 100,0 & 99,1 & 98,1 & $>0,05$ \\
\hline Disponibilidade de água diária & 94,2 & 95,3 & 95,5 & 88,8 & $>0,05$ \\
\hline Esgoto a "céu aberto" & 14,1 & 4,4 & 18,3 & 26,1 & $<0,001$ \\
\hline $\begin{array}{l}\text { Casa de alvenaria inacabada ou construída } \\
\text { com outro material (madeira, zinco, papelão) }\end{array}$ & 33,0 & 11,5 & 40,0 & 61,0 & $<0,001$ \\
\hline $\begin{array}{l}\text { Referência de criança com verminose } \\
\text { no domicílio }\end{array}$ & 6,8 & 0,8 & 5,6 & 19,5 & $<0,001$ \\
\hline
\end{tabular}

* Teste qui-quadrado;

** Valor referência do salário mínimo $=\mathrm{R} \$ 240,00$ (agosto/2003).

riada e multivariada, que estão apresentados nas Tabelas 3 e 4 .

Considerando as características do chefe da família, o fator mais importante foi a escolaridade. Chefes com escolaridade abaixo do nível de ensino fundamental, quando comparados aos demais acima deste nível, apresentaram 4,6 vezes mais chances de ter insegurança alimentar leve e 8,4 vezes mais de ter insegurança alimentar moderada e grave.
Cada criança menor de cinco anos de idade no domicílio representou uma razão de chances de 2,1 de a família ser classificada em insegurança alimentar moderada e grave e de 1,6 em insegurança alimentar leve, o mesmo ocorrendo com a ascensão do número de menores de 18 anos de idade.

A ausência de rede de esgoto mostrou elevar a chance de insegurança alimentar moderada e grave em 5,3 vezes e de insegurança alimentar leve em 1,9 vez. 
Análise da associação da insegurança alimentar leve e da insegurança alimentar moderada e grave e as demais condições sociais da família por meio de regressão logística politômica univariada. Campinas, São Paulo, Brasil, 2003.

\begin{tabular}{|c|c|c|c|c|c|c|}
\hline \multirow[t]{2}{*}{$\begin{array}{l}\text { Variável (categoria } \\
\text { de análise) }\end{array}$} & \multicolumn{3}{|c|}{$\begin{array}{c}\text { Insegurança alimentar leve vs. } \\
\text { segurança alimentar }\end{array}$} & \multicolumn{3}{|c|}{$\begin{array}{c}\text { Insegurança alimentar moderada e } \\
\text { grave vs. segurança alimentar }\end{array}$} \\
\hline & OR & IC95\% & Valor de $p$ & OR & IC95\% & Valor de $\mathrm{p}$ \\
\hline \multicolumn{7}{|l|}{ Sexo do chefe da família } \\
\hline Feminino vs. masculino & 1,23 & $0,76-1,98$ & 0,4014 & 1,30 & $0,74-2,29$ & 0,3628 \\
\hline \multicolumn{7}{|l|}{ Escolaridade do chefe da família } \\
\hline \multicolumn{7}{|c|}{ Analfabeto ou fundamental incompleto vs. } \\
\hline nível médio ou universitário & 4,60 & $2,50-8,49$ & 0,0009 & 8,40 & $4,07-17,35$ & 0,0001 \\
\hline \multicolumn{7}{|l|}{ Nível fundamental completo vs. nível } \\
\hline médio ou universitário & 3,21 & $1,90-5,42$ & 0,0011 & 3,30 & $1,65-6,59$ & 0,0067 \\
\hline \multicolumn{7}{|l|}{ Raça/cor auto-referida do informante } \\
\hline Preta vs. branca & 3,40 & $1,36-8,52$ & 0,0242 & 4,60 & $1,62-13,10$ & 0,0340 \\
\hline Parda vs. branca & 1,34 & $0,79-2,27$ & 0,5107 & 3,25 & $1,79-5,88$ & 0,0443 \\
\hline \multicolumn{7}{|l|}{ Renda mensal familiar (salário mínimo) } \\
\hline Até 2 vs. mais de 2 & 5,43 & $3,07-9,60$ & 0,0001 & 26,78 & $13,49-53,17$ & 0,0001 \\
\hline \multicolumn{7}{|c|}{ Família possui membro com nível universitário } \\
\hline Não vs. sim & 4,15 & $2,41-7,15$ & 0,0001 & 11,34 & $4,13-31,13$ & 0,0001 \\
\hline \multicolumn{7}{|l|}{ Família possui membro analfabeto } \\
\hline Sim vs. não & 2,34 & $0,90-6,04$ & 0,0798 & 5,63 & $2,18-14,58$ & 0,0004 \\
\hline \multicolumn{7}{|l|}{ Número de menores de 5 anos no domicílio } \\
\hline Acréscimo de um integrante & 1,56 & $1,13-2,16$ & 0,0075 & 2,10 & $1,45-3,04$ & 0,0001 \\
\hline \multicolumn{7}{|c|}{ Número de menores de 18 anos no domicílio } \\
\hline Acréscimo de um integrante & 1,72 & $1,31-2,25$ & 0,0001 & 2,31 & $1,71-3,13$ & 0,0001 \\
\hline \multicolumn{7}{|c|}{ Número de adultos no domicílio (18 a 64 anos) } \\
\hline Acréscimo de um integrante & 1,05 & $0,85-1,31$ & 0,6413 & 0,93 & $0,70-1,22$ & 0,5811 \\
\hline \multicolumn{7}{|l|}{ Número pessoas por cômodo do domicílio } \\
\hline Acréscimo de um integrante & 8,93 & $4,34-18,35$ & 0,0001 & 17,73 & $8,13-38,66$ & 0,0001 \\
\hline \multicolumn{7}{|l|}{ Esgoto da rede pública no domicílio } \\
\hline Não vs. sim & 1,94 & $1,00-3,76$ & 0,0499 & 5,19 & $2,65-10,17$ & 0,0001 \\
\hline \multicolumn{7}{|l|}{ Tipo de construção da moradia } \\
\hline Outro material vs. alvenaria acabada & 5,70 & $3,32-9,79$ & 0,0001 & 13,98 & $7,50-26,05$ & 0,0001 \\
\hline
\end{tabular}

* Teste qui-quadrado;

** Valor referência do salário mínimo $=\mathrm{R} \$ 240,00$ (agosto/2003).

No modelo final (Tabela 4), multivariado, o indicador que apresentou maior impacto na segurança alimentar foi a renda familiar mensal em salários mínimos; famílias com renda mensal inferior a dois salários mínimos tiveram quase 13 vezes mais chances de estar em insegurança alimentar moderada e grave e 2,9 vezes mais chances de estar em insegurança alimentar leve. Mesmo assim, controlando-se pelo efeito da renda, o número de pessoas por cômodo no domicílio e o tipo de moradia apresentaram associação significativa com a insegurança alimentar. A razão de chances de classificação na categoria insegurança alimentar moderada e grave foi 5,2 vezes maior para cada pessoa adicional por cô- modo; na categoria insegurança alimentar leve, a razão foi 3,6 vezes maior. Em relação à moradia inacabada e precária, a chance de ter insegurança alimentar moderada e grave foi de 3,5 e de ter insegurança alimentar leve foi de 2,9, ambos os casos em comparação à moradia de alvenaria acabada.

As outras variáveis não permaneceram no modelo final ajustado, mesmo que, isoladamente, apresentassem fortes associações com a insegurança alimentar moderada e grave e insegurança alimentar leve, medidas pelo valor do odds bruto. 
Análise da associação da insegurança alimentar leve e da insegurança alimentar moderada e grave e as demais condições sociais da família por meio de regressão logística politômica multivariada ( $\mathrm{n}=431$ ). Campinas, São Paulo, Brasil, 2003.

\begin{tabular}{|c|c|c|c|c|c|c|}
\hline \multirow[t]{2}{*}{$\begin{array}{l}\text { Variável (categoria } \\
\text { de análise) }\end{array}$} & \multicolumn{3}{|c|}{$\begin{array}{l}\text { Insegurança alimentar leve vs. } \\
\text { segurança alimentar }\end{array}$} & \multicolumn{3}{|c|}{$\begin{array}{c}\text { Insegurança alimentar moderada e } \\
\text { grave vs. segurança alimentar }\end{array}$} \\
\hline & OR & IC95\% & Valor de $p$ & OR & $\mathrm{IC} 95 \%$ & Valor de $p$ \\
\hline \multicolumn{7}{|l|}{ Renda mensal familiar (salário mínimo) } \\
\hline Até 2 vs. mais de 2 & 2,94 & $1,58-5,46$ & 0,0006 & 12,95 & $6,19-27,10$ & 0,0001 \\
\hline \multicolumn{7}{|c|}{ Número de pessoas por cômodo do domicílio } \\
\hline Acréscimo de um integrante & 3,55 & $1,59-7,93$ & 0,0020 & 5,19 & $2,19-12,30$ & 0,0002 \\
\hline \multicolumn{7}{|l|}{ Tipo de construção da moradia } \\
\hline Outro material vs. alvenaria acabada & 2,91 & $1,57-5,40$ & 0,0007 & 3,52 & $1,66-7,43$ & 0,0010 \\
\hline
\end{tabular}

\section{Discussão}

Por tratar-se de um estudo transversal não é possível afirmar relações de causalidade entre a variável dependente (insegurança alimentar) e as variáveis independentes.

Esta proposta de análise distinguiu-se da publicada pelo Departamento de Agricultura dos Estados Unidos, utilizada nos inquéritos americanos 13, de formar quatro categorias de segurança/insegurança alimentar, mas justifica-se, nesta população, por não se observar diferenças significativas relativas às variáveis independentes estudadas entre os grupos classificados em insegurança moderada e grave.

A insegurança alimentar em Campinas, assim como outros indicadores de qualidade de vida, têm distribuição desigual na população, fato que revela subgrupos mais vulneráveis às condições de vida desfavoráveis e reforça a situação de iniqüidade já enfaticamente relatada em estudos epidemiológicos 15 . As carências e privações sociais observadas são múltiplas e, segundo Drachler et al. 3, dependem muito mais de desigualdades estruturais que se mantêm ao longo do tempo do que de atributos individuais, ainda que aqui tenham sido dimensionadas a partir de unidades familiares.

Neste estudo, que observou basicamente a insegurança alimentar no âmbito da família, os atributos individuais associados a esse fenômeno podem estar refletindo o impacto das condições estruturais historicamente adversas. Entre eles, a cor da pele tem sido amplamente utilizada em estudos sobre desigualdades, apresentando a população de cor preta mais desvantagens no que se refere a condições de saúde, taxas de mortalidade, além de nível de educação, emprego e renda 16 . Vários estudos apontam maiores dife- renças sociais segundo raça/cor, sendo as prevalências sempre mais elevadas em pretos/pardos de baixa escolaridade, faixas salariais menores, desempregados, com trabalho informal e precário, vítimas de trabalho infantil e ocupações penosas $17,18,19$.

Nesta análise, aparece também a desigualdade na segurança alimentar. As pessoas de cor preta estão mais sujeitas à insegurança alimentar em comparação com as de cor parda; estas, por sua vez, mais do que as de cor branca. Tais diferenças não se mantiveram significativas na presença dos efeitos de renda, número de pessoa por cômodo e tipo de moradia.

O obscurecimento da questão racial como condicionante de desigualdades sociais tem sido observado em outros estudos epidemiológi$\cos 20,21$, merecendo, conseqüentemente, novas abordagens para seu esclarecimento, como, por exemplo, a utilização de um modelo de níveis múltiplos. Cabe postular que o fenômeno do branqueamento 22 tenha diminuído a força da associação entre insegurança alimentar e cor preta/parda, haja vista a relativa pequena proporção de domicílios em que o informante se autodeclara dessa cor, podendo, assim, o tamanho da amostra ter sido insuficiente para evidenciar as diferenças.

A insegurança alimentar moderada e grave concentra-se em famílias com maior número de membros, menor renda, maior aglomeração intradomiciliar, expostas a condição de saneamento básico precária e cujos responsáveis e membros têm menor escolaridade.

As variáveis, portanto, que melhor explicam a insegurança alimentar, em ambos os níveis, são renda abaixo de dois salários mínimos, moradia construída de forma precária e aglomeração intradomiciliar. 
Não é novidade, na nossa realidade social, a identificação de que parcela expressiva da população brasileira vive em condição de insegurança alimentar ou fome, do mesmo modo que não surpreende a sua distribuição desigual nos diferentes estratos sociais. A iniqüidade é relatada como originária do processo de colonização e vem se retroalimentando, de forma ininterrupta, até os dias de hoje 23 . Assim, esse problema, que já foi muito bem relatado por Castro, na década de 3024 , vem se mostrando como uma manifestação persistente do quadro de desigualdade ao longo de nossa história, ainda que, nas últimas décadas, em patamares mais baixos 25 .

Renda, neste estudo, assim como em outros, tem sido descrita como o determinante mais importante da insegurança alimentar e da fome, porém, segundo Rose ${ }^{26}$, não é um indicador suficiente, pois não considera muitas das diferenças existentes entre as famílias, nem os problemas particulares ou singulares próprios de cada família. Renda é, para esse e outros autores, um indicador indireto e não suficiente para medida da insegurança alimentar. Muitas famílias que se encontram acima da linha de pobreza podem apresentar insegurança alimentar, dependendo de outras condições que competem com o acesso aos alimentos. Nos Estados Unidos, Halminton et al. 27 , usando a linha de pobreza como ponto de corte, encontraram $17 \%$ de fome entre as famílias com renda menor que $50 \%$ da linha de pobreza e $1,4 \%$ de fome entre aquelas com renda maior que $185 \%$ da linha de pobreza. Nos anos de 1989 a 1991, nos Estados Unidos, Rose et al. 28 encontraram $16 \%$ de fome entre os que tinham renda menor que $50 \%$ da linha de pobreza e menos de $1 \%$ de fome entre os que tinham renda maior de $185 \%$ da linha de pobreza. Trata-se de um indicador muito pontual, que não mede as crises pelas quais as famílias passam em diversos momentos, que afetam a sua segurança alimentar.

É necessário também compreender os processos de mudanças econômicas mais recentes quando se interpreta a segurança alimentar. Estudo tipo caso-controle realizado nos Estados Unidos, comparando famílias com renda acima da linha de pobreza em insegurança alimentar leve e segurança alimentar e coletando fatores de exposição retrospectivos aos oito últimos meses do diagnóstico, observou que, daquelas que estavam em insegurança alimentar leve, 37,5\% relataram ter perdido emprego, ou ter ganho mais um membro na família, ou perdido o auxílio-alimentação; entre aqueles em segurança alimentar, esse percentual era de $16,5 \%$. Além disso, apenas uma pequena porcentagem de famílias em insegurança alimentar leve (5,3\%) conseguiu guardar dinheiro no ano que antecedeu o diagnóstico, evento que ocorreu para 22,9\% daquelas famílias em segurança alimentar 29. Olson et al. 30 também encontraram relação entre mudanças econômicas recentes na família e insegurança alimentar leve: esta foi mais prevalente no grupo que teve gastos altos e inesperados no último ano.

Esses estudos reforçam que medidas diretas da segurança alimentar, obtidas pela percepção dessa condição, que se dá por meio de instrumentos como aquele utilizado nesta pesquisa, a EBIA, produzem indicadores sensíveis a essa situação, independentes de outras condições sociais, como, por exemplo, a renda.

Existem fortes desigualdades na sociedade campineira, na qual a prevalência de insegurança alimentar atinge mais da metade da população urbanizada, e sua forma mais grave (insegurança alimentar moderada e grave), insegurança com restrição quantitativa de alimentos, está concentrada nos grupos de famílias de mais baixa renda, vivendo em condições precárias de moradia e com alta densidade demográfica intradomiciliar.

Considera-se que a medida direta de segurança alimentar e nutricional por meio da EBIA seja um importante indicador para monitoramento da iniqüidade, podendo complementar um conjunto de indicadores sociais ou, mesmo de forma isolada, identificar grupos com vulnerabilidade social.

O monitoramento da iniqüidade com uso da EBIA poderá auxiliar os gestores da política de combate à fome e à miséria, tanto na identificação dos grupos para os quais os recursos deverão ser prioritariamente dirigidos, quanto no estabelecimento de metas de cobertura populacional, possibilitando a avaliação das intervenções. 


\section{Resumo}

O objetivo deste trabalho foi investigar a associação da insegurança alimentar com algumas variáveis indicativas de desigualdades sociais, como renda, escolaridade, raça/cor, composição familiar, características da moradia e condições de saneamento. Para medida de segurança alimentar, aplicou-se o instrumento EBIA (Escala Brasileira de Insegurança Alimentar) a uma amostra de 456 famílias residentes em área urbana do Município de Campinas. Verificou-se que a insegurança alimentar concentrou-se em famílias com maior número de membros menores de 18 anos, vivendo em construções precárias e com alta aglomeração de moradores, sem rede de esgoto, de baixa renda (menos de dois salários mínimos), cujo responsável não freqüentou a escola, sem membros com nivel universitário e nas quais os informantes referiram ter cor da pele preta. Considera-se que a medida direta de segurança alimentar por meio da EBIA seja um importante indicador para monitoramento da iniqüidade, podendo complementar um conjunto de indicadores sociais ou, mesmo de forma isolada, identificar grupos com vulnerabilidade social.

Segurança Alimentar e Nutricional; Iniqüidade Social; Condições Sociais

\section{Colaboradores}

G. Panigassi elaborou e redigiu o artigo. A. M. SegallCorrêa e L. Marín-León colaboraram na análise dos dados, redação e revisão do artigo. R. Pérez-Escamilla, M. F. A. Sampaio e L. K. Maranha realizaram contribuições à versão final do artigo.

\section{Agradecimentos}

À Organização Pan-Americana da Saúde, Ministério da Saúde e Ministério do Desenvolvimento Social e Combate à Fome, pelo apoio financeiro, e à Fundação de Amparo à Pesquisa do Estado de São Paulo (FAPESP), pela bolsa de professor visitante.

\section{Referências}

1. Programa das Nações Unidas para o Desenvolvimento. Relatório do Desenvolvimento Humano 2006. Nova York: Organizações das Nações Unidas; 2006.

2. Akkari AJ. Desigualdades educativas estruturais no Brasil: entre estado, privatização e descentralização. Educação \& Sociedade 2001; 22:163-89.

3. Drachler ML, Côrtes SMY, Castro JD, Leite JCC. Proposta de metodologia para selecionar indicadores de desigualdade em saúde visando definir prioridades de políticas públicas no Brasil. Ciênc Saúde Coletiva 2003; 8:461-70.

4. Mackenbach JP, Kunst AE, Cavelaars EJM, Groenhof F, Geurts JJM. Socioeconomic inequalities in morbidity and mortality in Western Europe. The EU Working Group on Socioeconomic Inequalities in Health. Lancet 1997; 349:1655-9.
5. Barros RP, Henriques R, Mendonça R. Desigualdade e pobreza no Brasil: retrato de uma estabilidade inaceitável. Rev Bras Ciênc Saúde 2000; 15:123-42.

6. Moreira AS. Dinheiro no Brasil: um estudo comparativo do significado do dinheiro entre as regiões geográficas brasileiras. Estud Psicol (Natal) 2002; 7:379-87.

7. Perez-Escamilla R, Segall-Corrêa AM, Maranha LK, Sampaio MFA, Marín-León L, Panigassi G. An adapted version of the U.S. Department of Agriculture Food Insecurity Module is a valid tool for assessing household food insecurity in Campinas, Brazil. J Nutr 2004; 135:1923-8.

8. Coimbra Jr. CEA, Santos RV. Saúde, minorias e desigualdades: algumas teias de inter-relações, com ênfase nos povos indígenas no Brasil. Ciênc Saúde Coletiva 2000; 5:125-32. 
9. Valente FLS. Do combate à fome à segurança alimentar e nutricional: o direito à alimentação adequada. In: Valente FLS, organizador. O direito humano à alimentação: desafios e conquistas. São Paulo: Editora Cortez; 2002. p. 37-70.

10. Jyoti DF, Frongillo EA, Jones SJ. Food Insecurity affects school children's academic performance, weight gain and social skills. J Nutr 2005; 135:2831-9.

11. Casey P, Goolsby S, Berkowitz C, Frank D, Cook J, Cutts D, et al. Maternal depression, changing public assistance, food security, and child health status. Pediatrics 2004; 113:298-304.

12. Laraia BA, Siega-Riz AM, Gundersen C, Dole N. Psychosocial factors and socioeconomic indicators are associated with household food insecurity among pregnant women. J Nutr 2006; 136:177-82.

13. Bickel G, Nord M, Price C, Hamilton W, Cook J. Guide to measuring household food security: revised 2000. Alexandria: United States Department of Agriculture, Food and Nutrition Service; 2000.

14. Hosmer DW, Lemeshow S. Applied Logistic Regression. New York: John Wiley \& Sons Inc.; 1989.

15. Silva JB, Barros MBA. Epidemiologia e desigualdade: notas sobre a teoria e a história. Rev Panam Salud Pública 2002; 12:375-83.

16. Instituto Brasileiro de Geografia e Estatística. Síntese de indicadores sociais 2003. Rio de Janeiro: Instituto Brasileiro de Geografia e Estatística; 2004 (Estudos e Pesquisas. Informação Demográfica e Socioeconômica, 12).

17. Paixão M. Nada haver ou tudo a ver? Diálogos entre a questão do desenvolvimento econômico e das relações raciais no Brasil. In: Sicsú J, Paula LF, Michel R, organizadores. Novo desenvolvimentismo: um projeto nacional de crescimento com equidade social. Barueri: Manole Editorial/Fundação Konrad Adenauer; 2005. p. 301-27.

18. Programa das Nações Unidas para o Desenvolvimento. As desigualdades sócio-raciais. In: Organização das Nações Unidas, organizador. Relatório de desenvolvimento humano 2003. Nova York: Organização das Nações Unida; 2003. p. 54-83.

19. Lopes F. Para além da barreira dos números: desigualdades raciais e saúde. Cad Saúde Pública 2005; 21:1595-601.

20. Almeida-Filho N, Lessa I, Magalhaes L, Araújo MJ, Aquino E, Kawachi I, et al. Alcohol drinking patterns by gender, ethnicity, and social class in $\mathrm{Ba}-$ hia, Brazil. Rev Saúde Pública 2004; 38:45-54.
21. Lolio CA, Pereira JCR, Lotufo PA, Souza JMP. Hipertensão arterial e possíveis fatores de risco. Rev Saúde Pública 1993; 27:357-62.

22. Maio MC, Monteiro S, Chor D, Faerstein E, Lopes C. Cor/raça no Estudo Pró-Saúde: resultados comparativos de dois métodos de autoclassificação no Rio de Janeiro, Brasil. Cad Saúde Pública 2005; 21:171-80.

23. Maricato E. Metrópole, legislação e desigualdade. Estud Av 2003; 17:151-66.

24. Castro J. Geografia da fome. São Paulo: Editora Brasiliense; 1967.

25. Instituto de Pesquisa Econômica Aplicada/Secretaria de Estado de Direitos Humanos/Ministério das Relações Exteriores. A Segurança Alimentar e Nutricional e o Direito Humano à Alimentação no Brasil. Brasília: Instituto de Pesquisa Econômica Aplicada 2002.

26. Rose D. Economic determinants and dietary consequences of food insecurity in the United States. J Nutr 1999; 129 Suppl:517-20.

27. Hamilton W, Cook J, Thompson W, Buron L, Frongillo E, Olson C, Wehler C. Household food insecurity in the United States in 1995: summary report of the food security measurement project. Washington DC: United States Department of Agriculture; 1997.

28. Rose D, Habicht JP, Devaney B. Household Participation in the Food Stamp and WIC Programs Increases the Nutrient Intakes of Preschool Children. J Nutr 1998; 128:548-55.

29. Gundersen C, Gruber J. The Dynamic Determinants of Food Insufficiency In: Andrews M, Prell $\mathrm{M}$, editors. Second food security measurement and research conference. v. II. Washington DC: Food and Rural Economics Division, Economic Research Service, U.S. Department of Agriculture; 2001. p. 92-110. (Food Assistance and Nutrition Research Report, 11-1).

30. Olson CM, Rauschenbach BS, Frongillo Jr EA, Kendall A. Factors contributing to household food insecurity in a rural upstate New York county. Family Economics and Nutrition Review 1997; 10:2-17.

Recebido em 08/Mai/2007

Versão final reapresentada em 11/Mar/2008 Aprovado em 19/Mar/2008 